\title{
Four-Point Probe Electrical Measurements on p-n-p InP Structures
}

\author{
C. A. C. Sequeira and D. M. F. Santos \\ Department of Chemical and Biological Engineering, Instituto Superior Técnico, \\ Technical University of Lisbon (TU Lisbon), Avenida Rovisco Pais 1, 1049-001, Lisbon, Portugal
}

Received on 7 November, 2006

\begin{abstract}
The diffusion of zinc into n-type InP has been studied by four-point probe electrical measurements on homogeneously doped crystals at $750{ }^{\circ} \mathrm{C}$. The zinc carrier concentration in the diffused layer was approximately $3 \mathrm{x}$ $10^{18} \mathrm{~cm}^{-3}$ and its mobility was assumed to be about $40 \mathrm{~cm}^{2} \mathrm{~V}^{-1} \mathrm{~s}^{-1}$. It was observed that the concentration of free carriers throughout the entire diffused region is always less than the number of introduced impurity atoms. Possible reasons are discussed to explain the observed differences. Moreover this non-correlation phenomenon did provide substantial backing to the Hall Effect and $\mathrm{C}-\mathrm{V}$ measurements that are being carried out to further analyse the $\mathrm{Zn}$-InP diffused layer.
\end{abstract}

Keywords: Indium phosphide; Four-point probe technique; Zinc diffusion; Impurity profiles

\section{INTRODUCTION}

The properties of indium phosphide (InP) are typical of the majority of the semiconducting III-V compounds and particularly close in some aspects to those of GaAs [1,2]. Both materials have direct band gaps differing by about $0.1 \mathrm{eV}$ and exhibit similar values for the electron and hole masses, $m_{e}^{*}$ and $m_{h}^{*}$. Accordingly, after the manufacture of good single crystal InP, its suitability for use in solid state devices, previously produced using GaAs, was investigated. Initial interest focused mainly on electroluminescent devices followed by microwave devices. Subsequently, the work carried out falls into two major categories. Firstly, there has been the work of a fundamental physics nature which attempts to measure various parameters of the material particularly from an electronic point of view. These may be, for example, estimations of $m_{e}^{*}$ and $m_{h}^{*}$ and the shape of the band structure in general, as well as examinations of basic conduction mechanisms and electromagnetic effects. The second category contains work of a more applied nature connected with device manufacture. It is with this category that we are mainly concerned for the present times, together with some of the more relevant current-voltage measurements that are being carrying on.

This paper deals with four-point probe measurements, but before reporting it, it is appropriate to refer some relevant previous work on InP, discuss such work, and to conclude with the need to perform electrical and other studies. These aims basically stem on the scarcity and lack of cohesion within and between the previous experimental reports coupled with the fact that InP is potentially a very important and commercially viable product [3-10]. It is hoped that the next section would provide a clearer picture of the problems posed and of our decision to tackle them.

\section{PREVIOUS WORK ON INP}

In 1961, Goldstein [11] investigated the self-diffusion of both indium and phosphorus in InP, using radiotracer techniques. In both cases, he found a relationship in the form

$$
D=D_{0} \exp (-E / k T)
$$

where $D$ is the diffusion coefficient, $D_{0}$ the diffusion factor, $E$ the activation energy, $k$ the Boltzmann constant and $T$ the temperature.

The diffusion of phosphorus was much slower and had an associated activation energy, $E$, of $5.65 \mathrm{eV}, 2 \mathrm{eV}$ greater than that for indium. The opposite might be expected from a simple consideration of atomic sizes since the indium atom is the larger of the two. From his results, Goldstein concluded that self-diffusion in InP occurred by the migration of each species through its own sub-lattice. This migration was not observed [12].

The effect of radiative recombination from $\mathrm{p}-\mathrm{n}$ junctions formed in InP had been observed in the 1950's [13] but little interest was shown in such structures until the discovery of laser action in 1963 [14]. For high enough currents in a forward direction, emission was confined to a band in the infrared of 903-910 nm. As a continuation of this work, Weiser and his co researchers assessed the performance of such devices giving particular emphasis to the methods and conditions of manufacture [15]. At $750{ }^{\circ} \mathrm{C}$, they report a variation of the junction position $x_{j}$ with time in the form $x_{j} \propto t^{1 / 2}$, over periods from 15 minutes to 2 hours. This is compared with similar results in GaAs, and it is concluded that the impurity profile is unlikely to be "erfc" in form but similar to the profiles found in GaAs [2,16,17].

At roughly the same time as the above work, Chang and Casey [16] studied the diffusion of zinc into InP using radiotracer techniques. They used undoped n-type single crystals of [111] orientation with a carrier concentration of $7 \times 10^{16}$ $\mathrm{cm}^{-3}$ and mobility $3500 \mathrm{~cm}^{2} \mathrm{~V}^{-1} \mathrm{~s}^{-1}$.

Diffusions were done in the range $600{ }^{\circ} \mathrm{C}$ to $900{ }^{\circ} \mathrm{C}$ in 2 $\mathrm{cm}^{3}$ ampoules evacuated to $10^{-3}$ torr. They used a few milligrams of zinc and no additional phosphorus. The experimental profiles obtained were not of "erfc" form.

The authors [15] describe them as consisting of a surface region, in which the zinc concentration falls off rapidly, followed by a flattening out of the profile until a steep diffusion front is seen below $10^{19} \mathrm{~cm}^{-3}$. This steep front is compared to 
that observed for $\mathrm{Zn} / \mathrm{GaAs}$ diffusions. Despite the absence of any extra phosphorus in the ampoule, they observe only very slight surface deterioration of the crystals and also conclude that the smooth profiles are indicative of no surface alloying. The values of diffusion coefficient $D$ as a function of concentration $C$ were evaluated by the Matano method. No preliminary experiments to determine the time dependence of the profile penetration were described and hence the validity of using this method, which depends on a $t^{1 / 2}$ dependence, is not substantiated. The analysis showed $D$ to vary monotonically with $C$, reaching a value of $D_{\max }$ and then falling away sharply. A plot of solubility, $C_{0}$, versus $1 / T\left(\mathrm{~K}^{-1}\right)$ was a straight line agreeing with the expression $C=1.4 \times 10^{25} \exp (-0.92 / k T)$, and coinciding with a theoretical calculation of the solubility variation with temperature for InP. In brief, they conclude that the mechanism of diffusion of InP cannot be determined from their data alone, believing however that it will be similar to that operating in GaAs and GaP. This is a parallel mode diffusion with interstitial diffusion being dominant at high concentrations and temperatures [1,2,3,16-21].

Further work by Chang in 1964 [22] showed how the junction depth varied with time and temperature. He concludes, from the basis of similarity to GaAs work, that an interstitialsubstitutional mechanism is operative for zinc diffusion in InP and other III-V compounds. The paper gives very little information about the experimental conditions of diffusion.

In 1967, Kundukhov et al. [23] published work on the diffusion of cadmium and zinc into InP. They used two methods to produce profiles and compared the results with the tracer profiles of Chang and Casey [16]. However, they give no information about the material used for the diffusions except to state the background carrier concentrations. Neither do they give any idea of the quantities of zinc and cadmium used in each diffusion. For the case of zinc, by comparison with Chang and Casey's work [16], incomplete ionisation is indicated even at the junction itself. That is, the number of free acceptors does not equal the total number of zinc atoms anywhere in the diffused layer. An attempt to saturate a slice of InP with cadmium at $950{ }^{\circ} \mathrm{C}$ led to a maximum achievable hole concentration of $3-5 \times 10^{18} \mathrm{~cm}^{-3}$, as measured by Hall Effect techniques. Plots of $x_{j}$ versus $1 / T\left(\mathrm{~K}^{-1}\right)$ and $D$ versus $1 / T\left(\mathrm{~K}^{-1}\right)$ show a linear dependence for both zinc and cadmium. They discuss the change in junction depths observed for the case of extra phosphorus in terms of changes in vapour pressure, stating that the zinc and phosphorus react together to form $\mathrm{ZnP}_{2}$. No evidence is provided to confirm this reaction.

Ryzhikov et al. [24] in 1968 carried out studies into the electrical and electroluminescence properties of InP pn junctions. With both zinc and phosphorus in the ampoule, they claim that no appreciable diffusion of zinc occurred.

Further studies, using Hall Effect techniques, by Galvanov et al. [25], in 1969, with n-type InP of donor concentration $10^{16}-10^{17} \mathrm{~cm}^{-3}$ showed that the maximum hole density was less than $10^{19} \mathrm{~cm}^{-3}$ as compared to values of surface concentration $C_{0}$ of approximately $10^{21} \mathrm{~cm}^{-3}$. The authors explain this by considering the partial compensation of positive and negative zinc ions, the zinc diffusing in the form of acceptor, donor and neutral species.
Also in 1969, Arseni [26-28] published results for the diffusion of copper, gold and silver into InP, using radiotracer analysis. For the case of copper, he describes a profile of high surface concentration with an "erfc" form further into the crystal.

Previous work [29] on the diffusion of copper into InP indicated that very little indiffusion occurred, due to the formation of copper phosphide, $\mathrm{CuP}_{2}$, on the crystal surface. Arseni [26] does not make such observation. The experimental conditions required for the formation of the compound are not reported in the original work.

Colliver et al. [30] have investigated the use of InP in the production of a "three-level oscillator". They report considerable difficulty in making electrical contacts, especially to p-type material.

Many other researchers [31-34] have studied InP diffused layers until recently, but they did not bring too much advance to the $\mathrm{Zn} / \mathrm{InP}$ system.

The above discussion is an indication of the comparatively small amount of relevant information available about InP, with reference to diffusion and the production and operation of $p-n$ junction devices. Over the past 30 years, most of the published work has been from Russian, Chinese and Japanese origin, being difficult to compile and analyse.

The extent to which a comparison may be made between the various reports is further limited by a number of factors. Firstly, there are differences in the materials used. These occur in the mode of growth, cutting orientation, doping, carrier concentration, surface preparation, etc., and although all are important factors in the analysis of the diffusion, none of the authors state them in full. Many omit even the basic factors of orientation and doping species. Experimental conditions themselves are important, especially the quantities of components used and the volume of the ampoule. Again, although these factors have shown to have great effect on the final profiles and junctions, some reports do not state the amount of impurity source used. Equally, no particular set of experimenters has covered a wide enough range of diffusion parameters, for given crystals, to produce an overall picture of the effect of their variation.

As previously discussed [12], the most reliable method of producing impurity profiles is radiotracer analysis. This method was employed by Chang and Casey [16] who have produced perhaps the greatest amount of information by one set of authors to date. However, it is worthy of note that their results and discussion are based on the evidence of four profiles produced over a temperature range of $600{ }^{\circ} \mathrm{C}$ to $900{ }^{\circ} \mathrm{C}$. As stated, the method of calculating $D$ as a function of $C$ by Matano analysis was used but its validity not proven by experiment. In their discussion, the authors talk about equilibrium states existing with vapour pressure in the ampoule determined by zinc liquid phases. However, only occasionally was any sign of a liquid phase apparent on the surface, in the form of small "lumps". The importance of the ternary phase diagram for analysis of such a system is not mentioned, although their elemental zinc source is far from an equilibrium ternary source.

The latter work of Chang on junction measurements must 
be open to some doubt in view of Kundukhov's results [23] which indicate incomplete ionisation of the indiffused zinc even at the junction. However, at the same time it must be said that the Russian work cannot be accepted without reservation. The paper of Colliver et al. [30] shows that considerable difficulty may be expected in making electrical contacts to ptype InP and thus indicates that a certain amount of effort will be needed to produce good contacts for the evaluation of carrier concentrations. Despite all the stated reservations about past work on InP, several points of interest continually arise from it and require further investigation. These may be split into two groups; chemical and electrical phenomena $[8,20,33-$ 38]. From a chemical viewpoint, there are the observations of many of the researchers regarding the interaction of zinc and phosphorus. There are reports of the production of zinc phosphide, indium droplets, indium layers and surface alloying. Similar observations have been made in studies of the $\mathrm{Zn} / \mathrm{GaP}$ system. However, little evidence in support of the appearance of a particular element or compound is given. No attempt has been made, for the $\mathrm{Zn} / \mathrm{InP}$ system, to make use of a ternary phase diagram to explain "chemical" diffusion properties. Indeed no such diagram has yet, to our knowledge, been presented in the literature. Electrically, the main point which emerges is the difference between the concentrations of free carriers and zinc atoms in the diffused p-layers. Two sources $[23,25]$ quote a ratio between these two parameters of about one hundred. One of these sources [23] also suggests incomplete ionisation effects even at the junction. Compensation between donor and acceptor type carriers is used to explain the low holes' mobility reported in heavily doped InP.

In summary, the previous work on InP is not extensive and in most cases is inadequate to provide firm evidence of diffusion properties and mechanisms. Therefore, we decided to reinitiate our work on InP to overcome the appointed difficulties. We found that this research is particularly significant and timeliness not so under an academic point of view but also given the possible importance of this material in device manufacture.

Essentially, the aim of the entire work is to investigate some of the reported effects whilst, at the same time, producing a large amount of experimental data. This involves a large number of diffusions, with variations in such parameters as source type, source quantities, and diffusion time and temperature. The major techniques employed are radiotracer and p-n junction analysis. Surface effects are investigated using optical and electron microscopy. The results of this experimentation are discussed in terms of the ternary phase diagram which is assumed to exist for the In-P-Zn system. Electrical measurements are carried out on both homogeneously doped specimens and on those containing a zinc atom concentration gradient. Four-point probe, Hall Effect, and C-V measurements are applied. In the present paper, four-point probe measurements are reported and analysed [12,31,39]. Although this technique is a classic and standard technique for semiconductor characterisation, its importance has increased in the present times, as it is well documented in the open literature [40-50].

\section{FOUR-POINT PROBE TECHNIQUE}

This is one of the simplest forms of experiment which may be carried out in order to measure the electrical characteristics of a material. The voltage measured between two points on the surface of a specimen, for a known value of specimen current, together with certain geometrical factors, gives the surface and bulk resistivity of the material. The thickness of the specimen must be known to evaluate the bulk value. The use of four-point probes eliminates any problems caused by contact resistance. Although simple to perform, the technique suffers from difficulties in the analysis of experimental data. These difficulties arise mainly from geometrical considerations, with correction factors being necessary for non infinite specimens. In the analysis of a diffused layer, it is useful, as discussed in our previous paper [12], to use a serial sectioning technique [51]. The change in resistivity values found between one step and the next is a measure of the carrier concentration in the removed layer. Several researchers have formulated equations to produce this number of carriers. However, in order to use these equations, it is always necessary to have a knowledge of the carrier mobility and, if applicable, its variation with concentration. If such information is not available, the technique is seen to be somewhat limited in usefulness. Nevertheless, it was felt that such a technique would provide a good starting point and would at least provide information about various aspects of electrical measurement on p-type InP.

\section{EXPERIMENTAL PROCEDURE}

In the course of this investigation, (100) oriented InP wafers $500 \mu \mathrm{m}$ thick, with a carrier concentration of $6-10 \times 10^{16}$ $\mathrm{cm}^{-3}$, and polished to a mirror-like finish, were used. A diffusion specimen was a section of a wafer, about $1 \mathrm{~cm}^{2}$ in area. The wafers were subjected to a standard cleaning procedure (boiling for $10 \mathrm{~min}$ each in $80{ }^{\circ} \mathrm{C}$ trichloroethane, acetone, and methanol; etching for $2 \mathrm{~min}$ in $0.5 \% \mathrm{Br}$ methanol; multiple rinsing in methanol and chloroform). After the cleaning procedure the wafers were loaded into a clean quartz ampoule containing amounts of phosphorus and non-radioactive zinc which were known, from previous radiotracer experiments, to give a certain profile. In most cases the source contained $1 \mathrm{mg}$ each of zinc and phosphorus. The loaded ampoule was evacuated to less than $10^{-2}$ torr, and the diffusion took place in a three-zone furnace accurate to $\pm 1{ }^{o} \mathrm{C}$. The p-n junction was made visible by stain etching with $\mathrm{KOH}-\mathrm{K}_{4}\left[\mathrm{Fe}(\mathrm{CN})_{6}\right]-\mathrm{H}_{2} \mathrm{O}$ solution (1:2:6, $10 \mathrm{~s}$ under intense illumination) and measured with a light microscope. The junction depths obtained varied from about 10 to $160 \mu \mathrm{m}$. For a given set of experimental conditions, the junction lines follow the crystal contours; some of them are straight and flat, others are not flat, and some contain a small number of inclusions.

For the four-point probe technique, a standard resistivity test rig was employed. A small piece of In or In/Zn alloy was moulded around the end of a short length $(\sim 200 \mu \mathrm{m})$ of thin steel wire, and the process was repeated 4 times. The 
four wires/ probes, spaced at $650 \mu \mathrm{m}$ intervals, were properly positioned on top of the crystal, and electrical contact by local heating welded them to the specimen. The final contact was about 500-1000 $\mu \mathrm{m}$ across its width. The specimen rested on a slab of insulating ceramic and the probe head was raised or lowered onto the specimen by means of a screw and spring mechanism.

After diffusion, the crystal was trimmed around the edge to produce a good p-n-p structure. The surface was etched back to the required initial depth and rinsed well in acetone. Positioning on the rig was such as to place, as nearly as possible, the centre of the specimen under the probes, which are independently sprung in the measurement head.

Current to the specimen was from a stabilised power supply and the corresponding voltage across the specimen was measured on an "S.E." digital voltmeter. In general, a supply voltage of 15-30 V produced a current flow of several milliamps in the circuit, when the contacts were properly "formed". This process consists of a rapid switching of current flow direction at a fairly high value of supply voltage $(\sim 30 \mathrm{~V})$. The effect is to produce local heating around the contact point and to weld the contacts to the specimen. The extent of surface damage produced by this technique is small provided that care is taken with the magnitude of the applied voltage. After "forming", a steady value of specimen current is obtained and the current-voltage characteristic of the system is ohmic. With simple mechanical contact, an unsteady, non-ohmic, situation exists. For typical specimens in this work, a current of a few milliamps produced voltages on the order of the millivolt.

\section{EXPERIMENTAL RESULTS}

Several experiments were carried out on InP crystals having undergone standard diffusion of 30 minutes in ampoules at $10^{-4}$ torr and $750{ }^{\circ} \mathrm{C}$, containing about $0.77 \mathrm{mg}$ of zinc (and $1 \mathrm{mg}$ of phosphorus). The results, in the main, were inconsistent and poor in quality. The lack of reproducibility arose from several characteristics of the techniques. Firstly, there was the problem of "forming" the contacts exactly in the same manner on each specimen. On a few occasions a too high voltage supply led to the burning out of a contact point. Secondly, the sensitivity to geometrical changes led to some difficulty. The size of a standard specimen was less than $1 \mathrm{~cm}^{2}$ and this increased the importance of geometrical correction factors, leading in some instances to a wide variation in calculated resistivity values. The positioning of the probes was also critical; especially since a successive layer removal technique was being employed in most cases. A simple jig in the same place after each layer was removed.

Despite these problems, however, several runs proved quite successful with the depth measurements found by the use of a dial gauge. Analysis of the results was carried out using the method of Lamorte [31]. He considers the removal of layers, $\Delta x$, from a diffused region, $x$, with corresponding changes in conductance. He derives an equation for the carrier concentration $\bar{N}(x)$ in a given layer in terms of the measured resistivity before and after its removal. With diffused specimens, the p- $\mathrm{n}$ junction acts as an insulating boundary and thus the p-type layer in these samples can be studied independently of the remainder of the sample. At constant current $I$,

$$
\bar{N}(x)=\frac{I}{C_{F} e \mu(\bar{N}) \Delta x}\left[\frac{1}{V(x)}-\frac{1}{V(x+\Delta x)}\right]
$$

where $\mathrm{V}$ is a measured voltage, $\mu(\bar{N})$ is the carrier mobility at a concentration $\bar{N}$ and $C_{F}$ is a geometrical correction factor. For one particular specimen $\mathrm{X}, C_{F}$ was 4.0 and $\mu(\bar{N})$ was given a fixed value of $40 \mathrm{~cm}^{2} \mathrm{~V}^{-1} \mathrm{~s}^{-1}$. No mobilityconcentration data for p-type InP was available and this value was estimated from other researchers' results. It is really a mean value assumed constant throughout the diffused region. Hall Effect measurements carried out by the present authors provided several relevant parameters with good reproducibility, particularly on homogeneously doped samples, leading to carrier mobility values close to the value of $40 \mathrm{~cm}^{2} \mathrm{~V}^{-1} \mathrm{~s}^{-1}$ assumed in the present study. They also indicated well the general effects observed by the four point probe technique, demonstrating that this technique provide substantial backing to the Hall Effect results. A paper on this type of experimentation would be submitted soon to this Journal.

The profile of carriers $\bar{N}(x)$ for the specimen is plotted in Fig. 1 with a corresponding tracer profile for comparison. In this figure, $\mathrm{X}$ stands for a p-n junction homogeneously annealed with a final thickness of $200 \mu \mathrm{m}$; sample $X \sqrt{2}$ was the sample submitted to a tracer profile for 15 minutes with a $\sqrt{2}$ applied time factor. This approximation of assuming an "erfc" form, still is quite adequate for the purpose of comparison between atom and carrier levels. At all points, the carrier profile is below the tracer curve. A sharp peak $\left(\sim 10^{19} \mathrm{~cm}^{-3}\right)$ in carrier concentration near to the surface is followed in the bulk by a flat region of uniform concentration at about $3 \times 10^{18} \mathrm{~cm}^{-3}$. The pn junction, found by the chemical staining corresponds to a higher atomic concentration on the tracer profile.

The tabulated results for the four-point probe (sample X) experiment are shown in Table 1. Although no great accuracy can be attached to this and similar results, they do indicate a general trend for a considerable difference between atom and carrier concentrations. For diffusions carried out at 650, 700, 800,850 and $900{ }^{\circ} \mathrm{C}$ similar trends were observed, thus it was decided to interrupt the four-point probe measurements.

\section{DISCUSSION}

One important feature of the four-point probe results is the fact that, in a diffused layer, the number of electrically active carriers does not equal the total number of indiffused impurity atoms. The number of atoms is always the larger quantity. With this established, it is necessary to discuss the possible reasons for this effect.

The situation is one of an n-type semiconductor containing donors and electrons to which are added acceptors and holes by the indiffusion of impurity atoms. The concentrations of the various species involved are determined by Fermi-Dirac Statistics and one possible explanation of the above results 
TABLE I: Four-point probe measurements on the $X$ specimen.

\begin{tabular}{|l|l|l|l|l|l|l|l|l|}
\hline $\begin{array}{l}\text { Layer } \\
\text { no. }\end{array}$ & $\begin{array}{l}\text { Thickness } \\
\text { removed / side } \\
(\mu \mathrm{m})\end{array}$ & $\begin{array}{l}\text { Total thickness } \\
\text { removed } \\
(\mu \mathrm{m})\end{array}$ & $\begin{array}{l}\text { Voltage } \\
\left(10^{-3} \mathrm{~V}\right)\end{array}$ & $\begin{array}{l}1 / \mathrm{V} \\
\left(\mathrm{V}^{-1}\right)\end{array}$ & $\begin{array}{l}\text { Voltage } \\
\text { difference } \\
\left(\mathrm{V}^{-1}\right)\end{array}$ & $\begin{array}{l}1 / \text { layer } \\
\text { thickness } \\
\left(\mu \mathrm{m}^{-1}\right)\end{array}$ & $\begin{array}{l}\text { Carrier } \\
\text { concentration } \\
\left(10^{18} \mathrm{~cm}^{-3}\right)\end{array}$ & $\begin{array}{l}\text { Profile } \\
\text { penetration } \\
(\mu \mathrm{m})\end{array}$ \\
\hline Original surface & 2.10 & 2.10 & 14.60 & 68.49 & & & & \\
\hline 1 & 2.46 & 4.56 & 16.10 & 62.11 & 0.98 & 0.407 & 0.78 & 1.05 \\
\hline 2 & 2.35 & 6.91 & 18.80 & 53.19 & 8.92 & 0.426 & 7.41 & 3.3 \\
\hline 3 & 2.28 & 9.19 & 20.20 & 49.50 & 3.69 & 0.439 & 3.16 & 5.7 \\
\hline 4 & 2.28 & 11.47 & 21.60 & 46.29 & 3.21 & 0.439 & 2.75 & 8.0 \\
\hline 5 & 2.35 & 13.82 & 24.60 & 40.65 & 5.64 & 0.426 & 4.69 & 10.3 \\
\hline 6 & 1.87 & 15.69 & 27.50 & 36.36 & 4.29 & 0.535 & 4.48 & 12.6 \\
\hline 7 & 5.30 & 20.99 & 41.40 & 24.15 & 12.21 & 0.189 & 4.49 & 14.5 \\
\hline 8 & 5.52 & 26.51 & 79.80 & 12.53 & 9.62 & 0.181 & 3.49 & 18.4 \\
\hline 9 & 2.76 & 29.27 & 116.50 & 8.58 & 3.95 & 0.362 & 2.79 & 23.8 \\
\hline 10 & 2.54 & 31.81 & 190.00 & 5.03 & 3.55 & 0.394 & 2.72 & 28.0 \\
\hline 11 & 3.62 & 35.43 & unsteady & - & - & - & - & 30.6 \\
\hline 12 & 6.27 & 41.70 & 0.75 & & & & & 33.6 \\
\hline 13 &
\end{tabular}

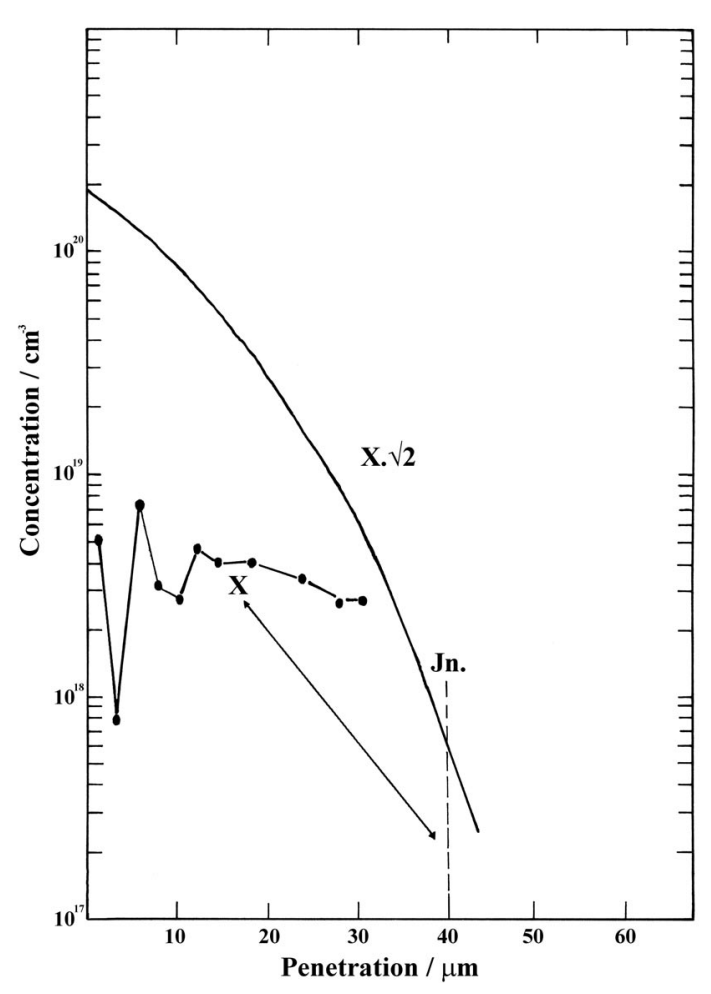

FIG. 1: Typical carrier profile determined from 4-point probe measurements and serial sectioning techniques. Also included are associated tracer profile, stained junction depth and background donor level.

arises purely from a statistical standpoint. As the impurity atoms are added to the crystal, the position of the Fermi-level will shift from its original level close to the conduction band towards the valence band. At moderate ptype doping, the Fermi-level is above the zinc impurity levels and these are therefore nearly all full. They have become full by accepting electrons from the donor levels and also from the valence band, leaving $N_{D}$ ionised donors and $\left(N_{A}-N_{D}\right)$ holes in the valence band. In fact, this is an approximation since not all of the donors and acceptors will become ionised and so the neutrality condition is actually,

$$
P=N_{A}^{-}-N_{D}^{+} \approx N_{A}-N_{D} \approx N_{A}\left(\operatorname{for}_{A} \gg N_{D}\right)
$$

However, for higher doping the Fermi-level approaches and then passes through the acceptor levels which then become less than a half full. Now, the approximation $P=N_{A}$ is quite wrong and in fact $P<N_{A}$.

In this context, we have carried out the calculation of the number of holes in the valence band, $\mathrm{p}$, for different doping levels, at $300 \mathrm{~K}$. We have used the standard Fermi-Dirac equation for the number of holes in the valence band at temperature $T$ :

$$
N_{V}=\int_{-\infty}^{E_{V}} \frac{4 \pi\left(2 m_{h}^{*} / h^{2}\right)^{3 / 2}\left(E_{V}-E\right)^{1 / 2} d E}{1+\exp \left(E_{F}-E\right) / k T}
$$

with the parameters,

$$
\begin{array}{ll}
E_{V}=-1.29 \mathrm{eV} & m_{e}^{*}=0.073 \mathrm{~m} \\
E_{C}=0 & m_{h}^{*}=0.4 \mathrm{~m} \\
E_{D}=-0.008 \mathrm{eV} & N_{D}=8 \times 10^{16} \mathrm{~cm}^{-3} \\
E_{A}=-1.265 \mathrm{eV} &
\end{array}
$$

A plot of holes in the valence band versus impurity concentration is reproduced in Fig. 2.

From the results of this work, the interesting range of hole concentrations is between $10^{18} \mathrm{~cm}^{-3}$ and $10^{19} \mathrm{~cm}^{-3}$. In these two levels, the ratio between atoms and holes can be seen to 
vary between about 2.5 and 10 . It seems, therefore, that this statistical effect is not sufficient to account for the experimentally observed ratios of typical value 25 , which were obtained by four-point probe measurements.

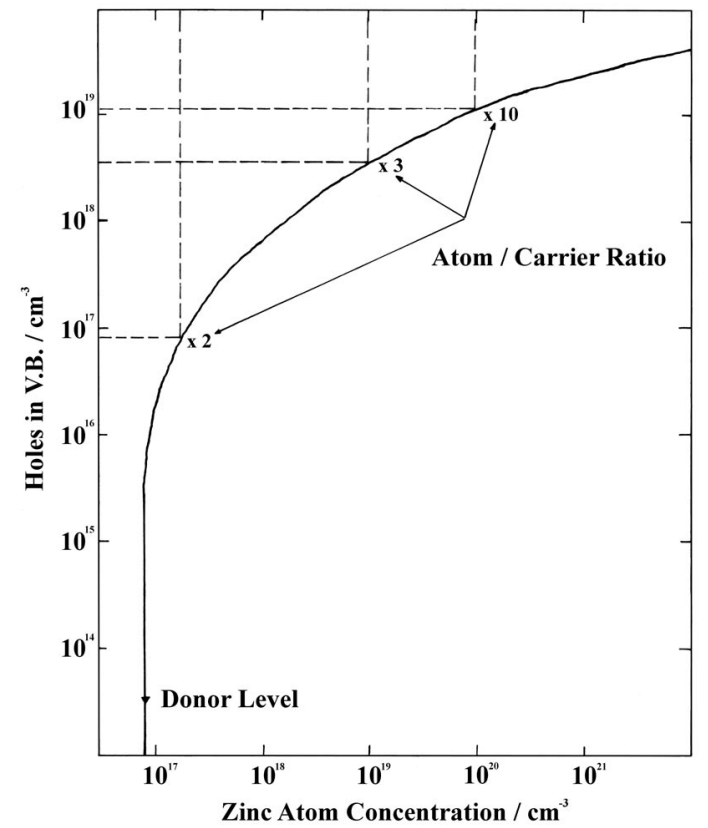

FIG. 2: A plot of the number of holes in the valence band versus the number of introduced impurity atoms, as calculated from FermiDirac Statistics, for zinc in n-type InP at $300 \mathrm{~K}$. It is assumed that the indiffused zinc atoms act as simple acceptors, residing on indium sites substitutionally.

Having established this possible cause of a discrepancy between atom and carrier concentrations, it is interesting to note that in the essentially similar $\mathrm{Zn} / \mathrm{GaAs}$ system there is a correspondence between the two quantities at much higher concentrations than expected from the simple statistical argument. This effect is possibly due to the appearance of impurity bands which complicates the statistics a good deal, modifying the density of states function for the valence band. The existence of these bands has been clearly shown in GaAs by the photoluminiscence work of Tuck [32]. Since there is no reason why similar bands should not occur in InP, it seems likely that the atom/carrier ratios quoted at given impurity levels from the simple statistics may actually be too high, leaving the statisti- cal explanation of experimental results as an even less likely possibility.

In the above arguments, it is assumed that all of the introduced zinc atoms act as simple acceptors, residing on indium sites substitutionally. In such a situation, one can equate the total number of atoms with $N_{A}$ and then compare the parameters $\mathrm{p}$ and $N_{A}$ to find an atom/carrier ratio. It is possible, however, that the observed difference between atom and carrier concentrations is due to a change in this situation. Then, the number of holes may actually be fairly close to the number of acceptors, as expected statistically, whilst being different of the number of impurity atoms. This difference in atom and acceptor concentrations may be considered in several ways. For instance, if more atoms than expected reside in interstitial positions, they will reduce the value of $P \approx N_{A}-N_{D}$ by acting as donors and compensating for some of the substitutional acceptors. It is also possible that the indiffused zinc may act as a neutral species to some extent. A grouping of atoms to form precipitates may lower their effective combined charge and thus restrict the number of associated free carriers. With the present experimental information, it is not possible to isolate any one of these theories, thus new ideas with a view to solving the problem are required and these impose the need for further experimental measurements and theoretical analyses. At present, manuscripts are being prepared on these matters, and soon will be submitted for publication.

\section{CONCLUSION}

This paper is devoted to experimental measurements of the doping rates, estimation of the diffusion parameters of atomic impurities and the relation between impurities doping rate and free carriers density. Experimental results obtained by fourpoint probe measurements for $\mathrm{Zn}$-InP diffused samples are compared with the related theoretical considerations which makes them feasible. The relevant feature of the results is the non equality found in the diffused layers between the number of electrically active carriers and the total number of indiffused zinc atoms. This observed difference in atom and carrier concentrations is analysed by several ways, namely by Fermi-Dirac Statistics, but the statistical effect is not sufficient to account for the experimental results. Other possible explanations are advanced, but further studies are necessary to determine the real nature of the zinc diffusion in InP.
[1] L. L. Chang and G. L. Pearson, J. Phys. Chem. Solids 25, 23 (1964).

[2] M. B. Panish and H. C. Casey, Jr., J. Phys. Chem. Solids 28, 1673 (1967).

[3] S. Aytac and A. Schlachetzki, Solid-State Electron. 24, 57 (1981).

[4] M. Syed and A. Siahmakoun, Opt. Mater. 27, 1629 (2005).

[5] N. Shamir, D. Ritter, and C. Cytermann, Solid-State Electron. 46, 785 (2002).
[6] L. Talzac, F. Barbarin, C. Varenne, L. Mazet, S. Pellier, and C. Soulier, Sensor. Actuat. B-Chem. 83, 149 (2002).

[7] Z. Hens and W. P. Gomes, Electrochim. Acta 43, 2577 (1998).

[8] P. Cova, A. Singh, A. Medina, and R. A. Masut, Solid-State Electron. 42, 477 (1998).

[9] A. Hooper, B. Tuck, and A. J. Baker, Solid-State Electron. 17, 531 (1974).

[10] J. W. Allen, J. Phys. Chem. Solids 15, 134 (1960).

[11] B. Goldstein, Phys. Rev. 121, 1305 (1961). 
[12] C. A. C. Sequeira and D. M. F. Santos, Czech J. Phys. 56, 549 (2006).

[13] R. Braunstein, Phys. Rev. 99, 1892 (1955).

[14] K. Wieser and R. S. Levitt, Appl. Phys. Lett. 1, 178 (1962).

[15] K. Wieser, R. S. Levitt, M. I. Nathan, G. Burns, and J. Weedall, Trans Met. Soc. AIME 230, 271 (1964).

[16] L. L. Chang and H. C. Casey, Jr., Solid-State Electron. 7, 481 (1964).

[17] M. Miczek, B. Adamowicz, and H. Hasegawa, Surf. Sci. 507, 240 (2002).

[18] St. J. Dixon-Warren, S. Zhang, R. Kuchibhatla, E. M. Griswold, A. Shen, F. Zheng, and S. R. Das, Thin Solid Films 472, 76 (2005).

[19] F. Schmidt and M. Mahnkopf, J. Cryst. Growth 138, 261 (1987).

[20] T. Kobayashi, K. Kurishima, and U. Gösele, J. Cryst. Growth 146, 533 (1995).

[21] M. Faur, Mi. Faur, D. J. Flood, and M. Goradia, Mat. Sci. \& Eng. B 28, 361 (1994).

[22] L. L. Chang, Solid-State Electron. 7, 858 (1964).

[23] R. M. Kundukhov, S. G. Metreveli, and N. V. Sincaev, Sov. Phys. Semicond. 1, 765 (1967).

[24] I. V. Ryzhikov, I. A. Novoselova, A. U. Kruchinin, and Yu. N. Nikolaev, Sov. Phys. Semicond. 2, 988 (1968).

[25] V. V. Galvanov, S. G. Metreveli, N. V. Sinkaev, and S. P. Staroseltseva, Sov. Phys. Semicond. 3, 94 (1969).

[26] K. A. Arseni, Sov. Phys. Solid-St. 10, 2263 (1968).

[27] K. A. Arseni, Sov. Phys. Semicond. 3, 1464 (1969).

[28] K. A. Arseni, Sov. Phys. Solid-St. 10, 2190 (1968).

[29] D. L. Kandall, Semiconductors and Semimetals Vol. 4 (Academic Press, New York, 1965).

[30] D. J. Colliver, C. Hilsum, J. R. Morgan, H. D. Rees, and B. Taylor, Inst. Phys. Phys. Soc. Conf. Ser. No. 9 (1971) p. 140.

[31] M. F. Lamorte, Solid-State Electron. 1, 164 (1960).

[32] B. Tuck, J. Phys. Chem. Solids 28, 1 (1967).

[33] R. Jakiela, A. Barez, E. Wegner, and A. Zagojski, Vacuum 78,
417 (2005)

[34] H. S. Shen, G.-Q. Yao, C.-C. He, K. Dwight, and A. Wold, Mater. Res. Bull. 23, 153 (1988).

[35] J. Decobert, D. Herrati, V. Colson, D. Leclerc, and L. Goldstein, J. Cryst. Growth 248, 390 (2003).

[36] A. Molassioti, F. Scholz, and Y. Gao, J. Cryst. Growth 102, 974 (1990).

[37] P. Franzosi, G. Salviati, M. Scaffardi, R. Andino, and F. Taiariol, J. Cryst. Growth 91, 90 (1988).

[38] A. E. Widmer and R. Fehlman, Solid-State Electron. 14, 423 (1971).

[39] L. J. Van der Pauw, Phillips Research Report 13, 1 (1958).

[40] P. J. Severin and H. Bulle, J. Electrochem. Soc. 122, 133 (1975).

[41] R. Kinder, L. Hulényi, and F. Mika, Phys. Status Solidi A 157, 393 (1996).

[42] C. L. Petersen, T. M. Hansen, P. Bøggild, A. Boisen, O. Hansen, T. Hassenkam, and F. Grey, Sensor. Actuat. APhys. 96, 53 (2002).

[43] S. Hasegawa and F. Grey, Surf. Sci. 500, 84 (2002).

[44] S. Hasegawa, I. Shiraki, F. Tanabe, R. Hobara, T. Kanagawa, T. Tanikawa, I. Matsuda, C. L. Petersen, T. M. Hansen, P. Bøggild, and F. Grey, Surf. Rev. Lett. 10, 963 (2003).

[45] A. P. Schuetze, W. Lewis, C. Brown, and W. J. Geerts, Am. J. Phys. 72, 149 (2004).

[46] D. C. Worledge, Appl. Phys. Lett. 84, 1695 (2004).

[47] Y. Ju, B.-F Ju, and M. Saka, Rev. Sci. Instrum. 76, 1 (2005).

[48] S. Dohn, K. Mølhave, and P. Bøggild, Sensor Letters 3, 300 (2005).

[49] B.-F Ju, Y. Ju, and M. Saka, J. Micromech. Microeng. 15, 2277 (2005).

[50] A. Ahaitouf, E. Losson, and A. Bath, Solid-State Electron. 44, 515 (2000).

[51] M. G. Beuhler, Stanford Research Report. SEL-66-064 (July 1966). 